\title{
Organisational Ontology Framework for Semantic Business Process Management
}

\author{
Agata Filipowska ${ }^{1}$, Martin Hepp ${ }^{2}$, Monika Kaczmarek ${ }^{1}$, Ivan Markovic ${ }^{3}$ \\ ${ }^{1}$ Department of Information Systems, Poznan University of Economics \\ 61-875 Poznan, Poland \\ \{a.filipowska,m.kaczmarek\}@kie.ue.poznan.pl \\ ${ }^{2}$ Chair of General Management and E-Business, Bundeswehr University Munich \\ 88579 Neubiberg, Germany \\ mhepp@computer.org \\ ${ }^{3}$ SAP Research \\ 76131 Karlsruhe, Germany \\ ivan.markovic@sap.com
}

\begin{abstract}
The field of Semantic Business Process Management (SBPM) has refuelled interest in using ontologies for the representation of the static and dynamic aspects of an enterprise and value chains. Putting the SBPM vision into practice, however, requires a consistent and operational network of ontologies reflecting the various spheres of enterprise structures and operations. Consistent means that the ontologies are based on compatible paradigms, have a compatible degree of detail, and include at least partial sets of alignment relations which allow data interoperability. Operational means that the ontology specifications are available in a single, current ontology formalism for which scalable repositories, reasoning support, APIs, and tools are available. In this paper, we describe a set of ontologies for SBPM that follows the mentioned requirements, and compare our work with the related efforts.
\end{abstract}

Keywords: ontology, Business Process Management, enterprise description, organisation framework

\section{Introduction}

Although BPM used together with SOA is believed to provide a complex approach to manage business processes in an enterprise, currently it offers only little support for automation of the BPM lifecycle. It is especially visible when it comes to the difficulties in smooth and automatic transition from one phase to another.

Various attempts were undertaken to provide a holistic view of the process space and achieve a higher level of automation in the BPM lifecycle. One of the most advanced initiatives in this area is the concept of Semantic Business Process Management (SBPM) developed in the SUPER project [1] taking advantage of the Semantic Web technologies (ontologies and reasoning mechanisms). 
In order to fulfil its aims, SBPM needs a semantic representation of various artefacts used within all of the phases of business process management. Therefore, apart from the semantic description of the process flow (control structure), the process content description is also required. The process content relates to the enterprise and its environment. Thus, putting the SBPM vision into practice requires a consistent and operational network of ontologies reflecting the various spheres of enterprise structures and operations. Consistent means that the ontologies are based on compatible paradigms, have a compatible degree of detail, and include at least partial sets of alignment relations which allow data interoperability. Operational means that the ontology specifications are available in a single, current ontology formalism for which scalable repositories, reasoning support, APIs, and tools are available.

The goal of this paper is to present the notion of the consistent and operational set of organisational ontologies aiming at providing vocabulary and constraints for describing the environment in which processes are carried out from the organisations perspective. In order to fulfil its aims, the article is structured as follows. First, the related work in the area of process artefacts representation is shortly discussed. In the following section the description of a consistent organisational ontology stack is given along with an application of the organisational ontologies within a domain-specific use case scenario. In Section 4 we conclude our work with a comparison between our work and related approaches and provide arguments in favour of our contribution.

\section{Related work}

The process content depends on organisation and its characteristics. Many researchers focused on development of models or ontologies cataloguing an appropriate scope of information on companies that should be considered when describing organizations or their processes e.g. [2][3]. For instance [2] highlighted that a process model should provide information on what is going to be done (i.e. functionality), who is going to do it (i.e. actors), when and where it will be done (i.e. location and time), how and why it will be done (i.e. means and motivation), as well as who depends on the results that are to be achieved (i.e. dependencies with other processes). Further on, the authors distinguished four different perspectives on the process, namely: functional, behavioural, organisational and informational. These perspectives underlie separate yet interrelated representations for analyzing and presenting processes.

The mentioned initiatives focused not only on the scope of information to be considered but also on its representation. Within the last few years there have been numerous initiatives attempting to capture the process-related and also organisationrelated information in a machine-friendly manner. Most of them focused on possible application of semantics, as ontologies are perceived as a good way to capture the domain and its relations $[4,5,10,15]$. These initiatives differ, when it comes to the scope of the process description they intend to cover, the level of details of the ontology created, as well as the formalism used.

One of the earliest initiatives was the TOVE project [6] that aimed at development of a set of integrated ontologies for modelling all kinds of enterprises (i.e. commercial and public ones) [4]. TOVE Common Sense Model of Enterprise included three 
levels: reference model with typical business functions (finance, sales, distribution, and administration), generic model (with such concepts as time, causality, space, etc.), and concept model (e.g. role, property). However, the granularity of developed ontologies may be perceived inconsistent what hampers their potential application..

The REA enterprise ontology [7] is based on elements of the REA (ResourceEvent-Actor) model [8]. The REA concepts and definitions are applied to the collaborative space between enterprises where the market exchange among trading partners occurs. Although REA is considered one of the most promising business domain ontologies, it is criticized for the lack of clarity and inconsistence e.g. [9].

The main aim of the e3-value project [10] was to propose the methodology to help in eliciting, analyzing, and evaluating e-commerce ideas. Therefore, the e3-value ontology was introduced as a tool to help explaining the concepts used to represent ecommerce ideas. The ontology provides concepts for describing economic exchange among partners. Other e3-value extensions like e3forces, e3competences should be of particular attention as they intend to model more advanced organisational aspects.

Enterprise Ontology (EO) [5] is a collection of terms and definitions relevant to business enterprises. It was developed as part of the Enterprise Project, with the aim to provide a framework for enterprise modeling. EO is divided into five parts: i) terms related to processes and planning, ii) terms related to organisational structure, iii) terms related to high-level planning, iv) terms relating to marketing and sales and v) terms used to define the terms of the ontology together with terms related to time. It was first completed in natural language format and then ported to Ontolingua [5].

Although significant effort was already committed to the creation of business and enterprise ontologies and the mentioned initiatives may provide an inspiration and foundation for developing organisational ontologies, to the best of our knowledge, there is no commonly accepted model that could be reused in various domains.

\section{Ontology framework}

The semantic process representation required by SBPM may be divided into three main groups, namely; process, organisational and domain-specific ontologies. Process ontologies [14] are created in order to describe the structure of a process, whereas organizational ontologies provide a description of artefacts, actors etc. that are utilised or involved in the process. The domain ontologies provide the additional information specific to an organisation from a given domain.

The organisational ontologies, this paper focuses on, aim at providing vocabulary and constraints for describing the environment in which processes are carried out from the organisations' perspective. Following [15], the organisational ontologies provide a basic vocabulary and structure for describing organisations, business goals and resources, define common types of divisions, roles and tasks, and define common types of business resources. Thus, the organisational ontologies layer provides a high level view on the organisation and process-related space and may be logically divided into a few subontologies, each of them describing different part of this space: 
1. Organisational Structure Ontology (OSO) focusing on organisational structure (hierarchy) of a company. It is designed as an upper level ontology and provides main structure and relations aiming at achieving domain independency.

2. Organisational Units Ontology (OUO) providing specification of typical units that may be found in a company. Along with Business Functions, Business Roles and Business Resources Ontologies, it provides extensions to OSO.

3. Business Roles Ontology (BROnt) representing roles in the organisation e.g. Designer, Process Modeller, IT Expert, CEO.

4. Business Functions Ontology (BFO) provides hierarchy of different functions that may be carried out within the company. It is supposed to enable vendor and domain independent classification of company processes and process fragments providing abstraction over single tasks constituting processes.

5. Business Resources Ontology (BRO) describing resources spent when carrying out certain processes or that may be results of certain task in a process.

6. Business Goals Ontology (BGO) modelling a hierarchy of business goals and provides a set of relations between them to enable goal-based reasoning.

In next sections of this paper each of the ontologies being a part of the organisational layer is presented. Due to the different character and status of the ontologies in question there is a difference in the level of details given while presenting them. They all have been modelled using WSML [25] in its Flight variant as the representation language.

\subsection{Organisational Structure Ontology and Organizational Units Ontology}

An organisational structure is defined as a hierarchy of an organisation showing how its elements work together in order to achieve organisation's goals. Following [17] the organisation structure encompasses: departments, employees, their responsibilities, resources etc. as well as relations among them.

The Organisational Structure Ontology (OSO) focuses on organisation hierarchy. The OSO structure benefited from already described models [4], [5], [12], [13] and [18]. The main distinguished concepts are: organisation, legal and non-legal entity, organisational unit, business function, person, skills, role and organisational position as well as resource (for exact definitions please refer to [16]).

It was designed as an upper level ontology providing the main domain-independent structure and relations. The structure of OSO makes it easy to be imported by other ontologies constituting the organisation ontology. And so Business Roles Ontology refers to the Role concept, Business Functions Ontology refers to the Business Function concept, Resource Ontology refers to the Resource, and finally Organisational Units Ontology refers to the Organisational Unit concept. OSO enables full description of an organisational structure and in addition links the mentioned organisational ontologies.

In turn, in case of the Organisational Units Ontology, an organisational unit is defined as any recognized association of people in the context of an enterprise. Following [17], it may be a corporation, a division, a department, a group or a team as well as a committee, task force, or a class [13]. 
As we decided to follow the OMG definition of Organisational Units [13], all units are divided into temporary and permanent ones. Temporary units are entities, that are created in order to carry out a task, project etc. They exist in an organisational structure only as long as a task is carried out. Permanent Units included in the structure are common for many organisations and were selected as a result of analysis of different organisational structures of existing companies as well as SAP Solution Maps [20]. A variety of existing departments as well as naming standards, forced us to use some simplification. In consequence, the developed ontology includes only the most common organisational departments in a production enterprise. Therefore, in order to describe some highly specific organisational units of e.g. security company, additional concepts need to be defined.

\subsection{Business Roles Ontology}

A business role is defined a set of expected behaviours, prerogatives and obligations featured by an actor [12]. The Business Roles Ontology provides a common meaning of concepts related to roles featured by organisational members. Each actor may play more than one role and these roles may change depending on the context.

The BRO was designed with an aim to provide a domain-independent, yet comprehensive, set of concepts, that would allow for description of roles in the organisation microenvironment i.e. both the inside structure of an organisation and its close surroundings. The proposed ontology allows to model both internal and external interactions and consists of 37 concepts. The fundamental concept for the ontology is a BusinessRole. The next level of the model splits up into three concepts. Two of them being specialized BusinessRole: InternalRole and ExternalRole. The third one InternalRoleType - provides the possibility to extend the description of any InternalRole. ExternalRole concept represents any BusinessRole that characterizes an agent from the outside of the organisation (e.g. BusinessPartnerRole further divided into IntermediaryRole and ProcurerRole). The assumption about ExternalRoles is that they are not disjoint. In contrast to ExternalRole, InternalRole is defined as BusinessRole that characterizes an agent from within the organisation (e.g. CustomerServiceRole). Any two InternalRoles are not mutually exclusive.

The current version of the Business Roles Ontology is supposed to answer the following exemplary competency questions: what role(s) does an actor or a group of actors play in the organisation? How many roles are featured by an actor or a group? Are there other actors with the same or similar role? What are the main and additional roles? Are the roles internal or external? Are there any specific (expanding) features characterizing a given role?

\subsection{Business Functions Ontology}

A business function is understood as a functional area of an enterprise e.g. Sales Management, Risk Management, Customer Management. The Business Functions Ontology (BFO) aims at standardizing meaning of business concepts and thus provides a common vocabulary for business functions within enterprises [18]. It was 
designed as a reference ontology, therefore, provides structure of business functions that are common for every company, regardless of the domain it operates in. It acts like an upper ontology for enterprise specific functional ontologies. It is supposed to be a starting point for further development of the business functions and should be extended with domain-specific functions.

Concepts included in the BFO as well as its structure are a result of analysis of existing ERP systems and abstracting over the SAP Business Maps [20]. While designing the BFO, the main goal was to provide as much information as possible and at the same time keep the ontology industry-independent.

BFO consists of two main structures [18], namely: Function and ActivityOrStep. The structure Function is the high level view on the functional side of the enterprise. It is specialised by such concepts as e.g.: Customer Management, Marketing Management and Sales Management. The Function structure contains only 40 concepts, 14 of them are top level, and the rest is grouped as their sub-concepts. The top level concepts name coherent and in some degree autonomous areas of functionalities. In addition, some of the top concepts such as e.g. FinanceManagement, are divided into sub-functionalities.

The ActivityOrStep structure supplements the Function structure and presents a more detailed view on the performed functions. An activity is understood as a unit of work identified within the business process e.g. UpdateSalesOrder. ActivityOrStep structure was designed at far more detailed level of abstraction and contains 920 concepts grouped as sub-concepts of 33 top-level concepts. Function and ActivityOrStep structures are connected via a transitive isSubPhaseOf attribute of each of top level concepts from ActivityOrStep sub-structure which determines which concept from Function structure is complemented by particular group of activities and steps. Appropriate axioms ensure the correctness of the specified relations.

The current version of the Business Functions Ontology is to answer the following competency questions: what are the main functional areas of the enterprise? how can the areas be further divided? what activities does the X-business function include? what kind of business function is an $\mathrm{X}$ - business sub-function? Within which functions the given activity or step is performed?

\subsection{Business Goals Ontology}

In our work we define business goal as the state of the enterprise that an action, or a given set of actions, is intended to achieve [24]. The Business Goals Ontology provides a standard set of properties and relations used in modelling a hierarchy of organisational business goals and enables formal verification of goal specifications.

Depending on the time horizon, business goals may be classified as operational or strategic. A strategic goal tends to be longer term and defined qualitatively rather than quantitatively. An operational goal is a short-term contribution to a strategic goal and provides the basis for measuring the progress toward meeting strategic goals.

Goals may be quantitative or qualitative. Quantitative goals are specified in a way allowing devising (measuring) the state of the goal by comparing values stated in the goal that express desired state of the world and the actual state. Qualitative goals are using textual descriptions of the desired state, and verification of the state of the goal 
requires human judgement. Quantitative goals need to have measures and threshold values defined, qualitative goals do not have to have such properties provided.

Business goal can have a more detailed description, it has an assigned measure for controlling the progress, deadline for completion and priority. In addition, as we can only control what we can measure [19], we assign a Measure to each quantitative goal. Each Measure has a defined Unit. Measure has a Current Value in the observed moment and a Desired Value which should be reached by a goal.

An important notion to keep in mind when talking about goals is time - goals have to be achieved in a certain time period (deadline). Goals can have priorities, which allow us to order the goal set according to their importance. Prioritization focuses attention on key areas, while allowing the modeler to describe goals which are currently perceived as less important or out of scope. Analysts can choose to show only those goals that are in scope. The goal hierarchy approach thus makes clear the e ects of scoping decisions, and allows trade-offs to be evaluated.

Business goal models usually contain a hierarchy of an organisation's business goals according to which the processes in the organisation are designed. The higher level goals are refined into more specific goals through AND or OR-decomposition, where the goals are decomposed into alternative subgoals (OR) or combinations of subgoals that must be satisfied (AND). The relation subgoal_of together with the constructs for AND/OR goal decomposition is used when creating the goal hierarchy. The relation subgoal_of is transitive, non-reflexive and anti-symmetric. An atomic goal is a goal that can not be further divided into subgoals.

Additional goal influencing relation types can exist. A support relationship between goals $g_{1}$ and $g_{2}$ suggests that achievement of goal $g_{2}$ assists achievement of goal $\mathrm{g}_{1}$; however achievement of goal $\mathrm{g}_{2}$ is not a necessary condition for achievement of goal $g_{1}$, or else goal $g_{2}$ would be a successor goal of goal $g_{1}$. On the other hand, a hinders relationship between goals $g_{1}$ and $g_{2}$ suggests that achievement of goal $g_{1}$ negatively influences achievement of goal $g_{2}$. Relations supports and hinders are transitive, non-reflexive and anti-symmetric. Goals can also conflict with each other if a goal $g_{1}$ hinders a goal of higher priority $g_{2}$, goals $g_{1}$ and $g_{2}$ are in conflict.

Introducing formalized business goal models enables new possibilities in process analysis [24]. For example the following queries can be executed using the business goals ontology: Show me all processes that support a specific goal. Show me the goal that is supported by the specific process. Show me all goals that are not completely specified. Filter goals on the basis of a given deadline and/or priority. Show me all goals that have no business process linked. Show me all processes that do not support any goal (gap analysis). Show me all processes that hinder the achievement of a specific goal. Show me all conflicting/redundant goals.

\subsection{Business Resource Ontology}

When formalizing knowledge about the business process space of an organisation, we also need a mean for describing the tangible and abstract resources that are relevant in the respective operations. For example, we need to know which tools are required for a particular production step. Therefore, we propose a Business Resources Ontology, which defines the core abstractions for resources, and associated notions of access, 
ownership, and consumption. This ontology can be imported and refined in more specific domain ontologies that define common types of business resources for specific domains, e.g. such things, e.g. WeldingStation or GalvanizationVessel.

We reuse the definition by Uschold et al, saying that a resource is ,something that can be used or consumed in an activity" [5, p. 41], as the most mature and conceptually clean specification of resources. Since being a resource is a role of an object and not a subtype of an object, the SUPER Resources Ontology does not contain a concept resource, but a relationship requiresAsResource.

This may be surprising but is a consequence of the fact that being a resource actually relates an object to an activity. In WSML syntax, this relation can be specified as follows: relation requiresAsResource (ofType Activity, ofType Object, of Type TypeOfPropertyRight, ofType _boolean); where:

- requiresAsResources means that the execution of the given Activity requires access in the form of the specified TypeOfPropertyRight to the specified Object;

- Activity is a subconcept of the AcitvityOrStep concept from the Business Function Ontology (i.e. bfo\#ActivityOrStep);

- Object is the explicitly identified or anonymous object needed. The characteristics of the object are specified by the respective class of object;

- TypeOfPropertyRight is the type of property right that is needed to specify what type of access is needed on that resource. For example, we may want to specify whether the object is modified, transformed, or even destroyed when serving as a resource, or whether it is just temporarily used. Economic theory and law uses the notion of property rights for distinguishing such types of access to a good. We use the four core distinctions from Property Rights theory. When we want to check whether a particular resource is available, we need a mean to specify what types of property rights are assigned to the entity who wants to use the respective object. This can be done best using a ternary relation hasPropertyRightOnObject (ofType Entity, ofType Object, ofType TypeOfPropertyRight);

- the boolean attribute indicates whether using the resource for the given activity consumes the resource or does not consume it. This is an important distinction, since some resources are lasting (e.g. documents, machinery) while others are consumed in the usage (e.g. coal, fuel).

Since we have no concepts for resources, the characteristics of an object serving as a resource must be reflected by the definition of the object and not its resource role. The following competency questions describe the scope of this subontology: which resources/types of resources exist in an enterprise? Which resources are required for a particular task? Which individual or legal entity has property rights on a particular resource? Does the regular usage of a given resource consume this resource?

\subsection{Use Case Study}

This section provides an example of application of organisational ontologies within the SUPER project based on a Digital Asset Management use case scenario in the telecommunications domain. The aim of this scenario is the provision of digital content to end users. The service provider, based on a customer query on the digital content specifying also the type of digital rights license the user is interested in, 
searches for the relevant digital content by several content providers. The search results are then presented to the user, who selects the content he wants to preview or purchase.

Telco ontologies used in this scenario were created to extend the organisational ontologies and provide domain specific concepts which are supplementary to the basic domain-independent concepts, while following the design principles provided by the organisational ontology layer. Further details on the development of telco ontologies within the SUPER project are provided in [22].

In the following, we provide some example queries which demonstrate the usage of our ontology framework within the use case scenario. The queries are expressed using WSML-Flight logical expressions, where boldfaced keywords refer to keywords in the WSML language.

Query 1. Show me all business goals with high priority:

?goal[bgo\#haspriority hasvalue high] memberof

bgo\#BusinessGoal

Query 2. Show me all business goals in conflict with the goal ImproveServices: bgo\#hinders (?g, Improveservices) and

bgo\#priority_lt(?g, Improveservices)

The following example demonstrates how we use our integrated view on the process space to query different process perspectives:

Query 3. Show me all processes which have Business Function related to 'Fulfilment' and use system 'CRM' as the Business Resource:

?process [bpo\#hasBusinessfunction hasvalue ?bf and bpo\#hasBusinessResource hasValue ?bres ] memberof bpo\#Process and ?bf memberof telbfo\#Fulfilment and ?bres memberof telbro\#CRM

Notice that bpo refers to the previously mentioned process ontology. Symbols telbfo and telbro denote the telco domain specific extensions of the Business Functions Ontology and Business Resource Ontology, respectively.

Query 4. Within which functions the activity ValidateOrder is performed? ?function[telbfo\#isSubPhaseof hasvalue Validateorder] memberof telbfo\#Businessfunction

Note that in answering this query we utilize the transitivity axiom of the is SubPhase Of relation.

Query 5. Show me all processes that support the business goal IncreaseRevenue:

Here we first need to find which business goals support the goal IncreaseRevenue: telbgo\#supports(?g, IncreaseRevenue)

where symbol telbgo represents the telco domain extension of the Business Goals Ontology. In order to answer our query we need to find processes which are annotated with the supporting business goal $g$ :

?process [bpo\#hasBusinessGoal hasvalue g] memberof

bpo\#Process 
Querying of organisational ontologies has been successfully utilized in various scenarios within the use case, such as: decision making support, reuse of business artefacts in process modelling and process redesign, see $[23,24,26]$ for more details.

\section{Discussion and conclusions}

In this section we compare the organisational ontologies presented in this article with the previous attempts in this area taking into account the defined criteria, namely: comprehensiveness, consistency and operational aspects (see Table 1).

\begin{tabular}{|c|c|c|c|c|c|}
\hline & \multicolumn{2}{|l|}{ Comprehensiveness } & \multirow{2}{*}{\begin{tabular}{|l} 
Consistency \\
\end{tabular}} & \multicolumn{2}{|c|}{$\begin{array}{l}\text { Operational } \\
\text { aspects }\end{array}$} \\
\hline Approach & Domain covered & 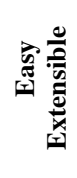 & & 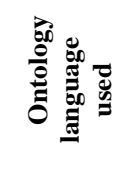 & 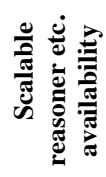 \\
\hline TOVE & $\begin{array}{l}\text { Generic enterprise } \\
\text { (Conceptualizations of: agents, roles, } \\
\text { positions, goals, communication, authority, } \\
\text { commitment thus it models an } \\
\text { organisational structure, activities and } \\
\text { management.) }\end{array}$ & - & - & $\begin{array}{l}\text { FOL } \\
\text { representa } \\
\text { tion }\end{array}$ & $+/-$ \\
\hline REA & $\begin{array}{l}\text { Creation of transfer of economic value. The } \\
\text { core concepts are: Resource, Event, and } \\
\text { Actor. }\end{array}$ & - & - & $\begin{array}{c}\text { None } \\
\text { commonly } \\
\text { accepted }\end{array}$ & - \\
\hline e3-value & $\begin{array}{l}\text { Identifying exchange of value objects } \\
\text { between the business actors. The basic } \\
\text { concepts in e3-value are actors, value } \\
\text { objects, value ports, value interfaces, value } \\
\text { activities and value exchanges. }\end{array}$ & $+/-$ & $+/-$ & $\begin{array}{l}\text { MODEL } \\
\text { descriptio } \\
\mathrm{n} \quad \text { logic } \\
\text { language }\end{array}$ & $+/-$ \\
\hline $\begin{array}{l}\text { Enterprise } \\
\text { Ontology }\end{array}$ & $\begin{array}{l}\text { Organisational structure, activities and } \\
\text { management. Four subject areas are } \\
\text { distinguished: activities and planning, } \\
\text { organisation, strategy, marketing }\end{array}$ & $+/-$ & + & $\begin{array}{l}\text { Informal } \\
\text { (text) and } \\
\text { semi- } \\
\text { formal } \\
\text { (Ontoling } \\
\text { ua) }\end{array}$ & - \\
\hline SUPER & $\begin{array}{l}\text { Organisation context with main focus on } \\
\text { functions, goals, organisation structure, } \\
\text { roles, resources. }\end{array}$ & + & + & WSML & + \\
\hline
\end{tabular}

Table 1. Comparison of the approaches

The approaches have usually different focus and the domain covered is not always comparable. While the TOVE and EO projects focus on providing generic enterprise model, REA and e3 value focus more on creation and transfer of economic value, our approach aims at describing organisation context relevant for the needs of business process modelling. The already developed ontologies cover often a substantial area of knowledge, however their usefulness is sometimes limited as the modelled parts of information space are defined on the different level of details and are sometimes 
inconsistent. In addition, only few initiatives define alignments between various ontologies constituting the set. For instance in TOVE project, three distinguished layers were designed at an inconsistent level of details (inconsistent granularity) and in case of REA the terminology for constructs is criticized for the lack of clarity [9]. The set of ontologies proposed for the needs of SUPER has been aligned and constitutes a consistent and coherent stack, as we have shown earlier in this paper.

In addition, the proposed approaches are rather domain-independent and general in essence, thus in order to improve their usability they should be considerably extended with a domain-specific vocabulary what sometimes is impossible as only few of them were designed bearing in mind further extension. In opposition to these approaches, our ontology stack was designed with the aim to achieve domain-independence as well as allow for easy extensions and usage of domain and industry specific ontologies within the framework as presented in the previous section.

Moreover, the lack of the formal version (expressed using one of the ontology languages) hampers practical application of some of the already defined ontologies (e.g. EO). Even if the ontology are presented in a more formal way (e.g. TOVE), very often the results are not serialized in any contemporarily recognized ontology language standard for which an efficient reasoner exists. In our case, usage of WSML formalism allows for application of a very good operational infrastructure. E.g. the currently available version of WSML2Reasoner uses the IRIS reasoner as a back-end reasoning engine. [21] reports that the functionality and performance of IRIS compare favourably with similar systems.

In this paper we have presented an organisational ontology framework for SBPM which integrates different views of an enterprise. Our ontology framework is designed with consistent level of detail and represented using a current ontology language for which scalable reasoners, repositories and tools are available. The framework has been successfully applied to a use case scenario in the telecommunication domain. By fulfilling the identified criteria to a greater extent in comparison to other approaches, the proposed organisational ontology framework has a great opportunity to become a useful and flexible tool to be applied in real world cases within different domains. With semantic annotation of business processes using organisational ontologies we also enable new types of business process verification and validation techniques.

\section{References}

1. SUPER project, http://www.ip-super.org

2. Curtis, B.; Kellner, M.I.; Over, J.: "Process Modeling". In Communications of the ACM, 35(9), Sep. 1992; pages 75-90.

3. Jablonski, St.; Bussler, Ch.: "Workflow Management - Modeling Concepts, Architecture and Implementation". International Thomson Computer Press, London, 1996.

4. Fox, Marc S.; Barbuceanu, Mihai; Gruninger, Michael; Lin, Jinxin: An Organisation Ontology for Enterprise Modeling. In: M. Prietula; K. Carley; L. Gasser (Hrsg.): Simulating Organizations: Computational Models of Institutions and Groups. AAAI/MIT Press, Menlo Park CA 1998.

5. Uschold M, King M, Moralee S, Zorgios Y (1998) The Enterprise Ontology. The Knowledge Engineer Review 13(1). 
6. Fox M (1992) The TOVE Project: A common-sense model of the enterprise. In: F. Belli, F. Radermacher (eds.) Industrial and Engineering Applications of Artificial Intelligence and Expert Systems. LNAI 604, Springer-Verlag, Berlin, pp 25-34.

7. Geerts, G. and McCarthy, W. E., An Accounting Object Infrastructure For KnowledgeBased Enterprise Models. IEEE Intelligent Systems \& Their Applications (August 1999)

8. McCarthy W. E., "The REA Accounting Model: A Generalized Framework for Accounting Systems in a Shared Data Environment”, The Accounting Review 1982.,

9. Lampe J.C. (2002) Discussion of an ontological analysis of the economic primitives of the extended-REA enterprise information architecture. International Journal of Accounting Information Systems, 3, 1 (March)

10. Gordijn, J., E3value in a Nutshell. Technical report, HEC University Lausanne, 2002.

11. SEKT Consortium, "PROTON Ontology," available at http://proton.semanticweb.org/, retrieved January 15, 2007, http://www.dip.semanticweb.org.

12. Mühlen, M. z., Evaluation of Workflow Management Systems Using Meta Models. In Proceedings of the Thirty-Second Annual Hawaii international Conference on System Sciences-Volume 5 - Volume 5 (January 05 - 08, 1999). HICSS. IEEE Computer Society.

13. OMG Document bmi/2006-11-02; Organization Structure Metamodel (OSM); 2nd Initial Submission; In Response to: Organization Structure Metamodel RFP (OMG Document bei/2004-06-05); Version 0.5 - November 9, 2006.

14. D.1.1. Business Process Ontology Framework, SUPER Deliverable, April 2007

15. Hepp, M., Roman, D.: An Ontology Framework for Semantic Business Process Management Proceedings of Wirtschaftsinformatik 2007, Karlsruhe, 2007

16. Abramowicz, W. et al., Organization Structure Description for the Needs of Semantic Business Process Management, Conference Proceedings of SBPM 2008

17. Malone, Thomas W. \& Sloan School of Management., 1986. "A formal model of organizational structure and its use in predicting effects of information technology," Working papers 1849-86., MIT, Sloan School of Management.

18. Abramowicz, W., et al., Semantic Enterprise Description for the Needs of Business Process Automation, Conference Proceedings of 1st IEEE International Workshop on Semantics for Business Process Management (SemBPM 2008)

19. DeMarco, T., Controlling Software Projects: Management, Measurement \& Estimation. Yourdon Press, New York, 1982.

20. SAP Business Maps, http://www.sap.com/solutions/businessmaps/index.epx

21. Bishop, B and Fischer, F., IRIS - Integrated Rule Inference System, Proceedings of the 1st Workshop on Advancing Reasoning on the Web: Scalability and Commonsense (ARea2008) hosted by the 5th European Semantic Web Conference (ESWC-08), 2008

22. D8.1. Telecoms Vertical Domain and Process Ontology, SUPER deliverable, 2007.

23. Markovic, I, Advanced Querying and Reasoning on Business Process Models, Proceedings of the 11th International Conference on Business Information Systems (BIS), Innsbruck, Austria, May 2008.

24. Markovic, I, Kowalkiewicz, M, Linking Business Goals to Process Models in Semantic Business Process Modeling, Proceedings of the 12th IEEE International EDOC Conference, Munich, Germany, September 2008.

25. Web Service Modelling Language (WSML), WSMO Working Group Final Draft, 2005.

26. Born, M, Filipowska A, Kaczmarek, M, Markovic, I, Business Functions Ontology and its Application in Semantic Business Process Modelling. Proceedings of the 19th Australasian Conference on Information Systems, 2008. 